\title{
Desintoxicación de dosis elevadas de metadona en una Unidad de Desintoxicación Hospitalaria (UDH). Experiencia clínica
}

\author{
Enrioueta Ochoa*; Estrella Salvador*; Agustín Madoz-Gúrpide**; Mar Lázaro* \\ * Servicio de Psiquiatría. Hospital Universitario Ramón y Cajal. Madrid. \\ **Centro de Salud de Mental San Blas. Madrid. \\ Enviar correspondencia a: \\ Enriqueta Ochoa. Servicio de Psiquiatría. Hospital Universitario Ramón y Cajal. Ctra Colmenar Km 9, 100. 28034 Madrid. \\ e-mail: eochoa.hrc@salud.madrid.org
}

Recibido: Septiembre de 2007

Aceptado: Marzo de 2008

\section{RESUMEN}

El aumento de adictos a opiáceos en tratamiento en metadona, junto con la mayor supervivencia de los pacientes VIH, ha supuesto en nuestro medio un incremento de la demanda de desintoxicación de dicha sustancia. Es práctica habitual en las Unidades de Desintoxicación Hospitalaria (UDH) solicitar una dosis máxima de metadona (alrededor de $40 \mathrm{mg}$ ) al ingreso para realizar la desintoxicación. Sin embargo, esto no siempre es posible, bien por el tiempo necesario para la disminución gradual ambulatoria, por aparecer sintomatología abstinencial, o por partir el paciente de dosis muy elevadas de metadona.

Se muestra la experiencia en 22 pacientes que en los últimos dos años realizan desintoxicación de metadona sin solicitar a los centros remitentes dosis límite máxima para su ingreso. Reciben durante la desintoxicación tratamiento con clonidina y benzodiacepinas (ajustada la dosis de éstos fármacos según el consumo concomitante de alcohol, benzodiacepinas o cocaína) y analgesia no opiácea. Al séptimo día se administra $50 \mathrm{mg}$ de naltrexona. De estos adictos, 21 realizaron la desintoxicación de forma adecuada.

Palabras clave: desintoxicación de metadona, adicción, opiáceos.

\section{ABSTRACT}

The increase in opiate addicts in treatment with methadone, coupled with improved survival of HIV patients, has meant an increase in the demand for detoxification from this substance in our environment. It is common practice in hospital detoxification units to request a maximum dose of methadone (around 40 mg) on beginning detoxification treatment. However this is not always possible, due to the time needed for a gradual decrease for outpatients making daily visits to the methadone dispensing centres, due to the appearance of withdrawal symptoms, or because the patient starts out from very high doses of methadone.

Reported here is an experience with 22 inpatients who over the last two years underwent detoxification from methadone without requesting from their treatment centres a maximum-limit dose at the start of their treatment. During the detoxification they are given treatment with clonidine and benzodiazepines (dosage being adjusted according to concomitant use of alcohol, benzodiazepines and cocaine) and non-opiate-based painkillers. On the seventh day they are given $50 \mathrm{mg}$ of naltrexone. Of these addicts, 21 completed the detoxification adequately.

Key words: detoxification from methadone, addiction, opiates

\section{INTRODUCCIÓN}

$E^{2}$ aumento de adictos a opiáceos en programas de mantenimiento con metadona (PMM), junto con la mayor supervivencia de los pacientes VIH en la última década, ha supuesto en nuestro medio la aparición de la demanda de desintoxicación a dicha sustancia.

En la Comunidad de Madrid, según datos de su memoria de 19981, se encontraban a final de ese año,
5431 pacientes en programas de mantenimiento con metadona (PMM), existiendo un incremento de plazas del 200\% sobre el año anterior, con una larga lista de espera generada por las pocas plazas existentes hasta ese momento.

La memoria del $2005^{2}$ informa que desde que se creó el registro de metadona en la Comunidad de Madrid, el total de pacientes que han sido incluidos en programas con agonistas opiáceos en esta Comunidad, han sido de 20305 pacientes, de los cuales 
permanecen en el programa 11171. De ellos 956 llevaban menos de 1 año, 1279 entre 1-2 años, 1480 entre 2-3 años, 1819 entre 3-4 años, 1395 entre 4-5 años, 1131 entre 5-6 años, 867 entre 6-7 años, 941 entre 7-8 años, 739 entre 8-9 años, 248 entre 9-10 años, 151 entre 10-11 años, 70 entre 11-12 años, 30 entre 12-13 años y 41 más de 13 años. Los motivos de salida del programa en estos años han sido variados, y entre ellos fueron por fin de tratamiento 1471 y por desintoxicación 225.

Ha sido en estos últimos años cuando se nos empieza a solicitar el realizar desintoxicaciones de metadona, pues hasta hace pocos años la escasez de plazas en nuestro medio y la mortalidad asociada al VIH hacían que la solicitud de desintoxicaciones fueran verdaderas rarezas.

La pauta habitual para realizar la desintoxicación de metadona ha sido una disminución gradual de la dosis del agonista de forma muy lenta a nivel ambulatorio, como se ha preconizado de forma clásica, con una disminución del 3\% semanal ${ }^{3}$. En general se recomienda una reducción gradual del $10 \%$ de la dosis al día como máximo, aunque conviene realizarla con más lentitud (1-5 mg/semana). En ocasiones puede retirarse más bruscamente y aplicar una pauta de desintoxicación similar a la de heroína (alfa2-agonistas, dextropropoxifeno, etc.) pero más prolongada en el tiempo y siempre dependiendo de la dosis de metadona que reciba el sujeto. Otra opción consiste en la desintoxicación hospitalaria con deprivación brusca de metadona y tratamiento sintomático del SAO durante 15 días $^{4}$. El intervalo de tiempo preciso para realizar la desintoxicación ambulatoria, en ocasiones es muy largo, sobre todo si se parte de dosis elevadas.

Es común observar que al ir reduciendo la dosis de metadona, partamos de la dosis que sea, y lo realicemos a la velocidad que sea, al llegar a dosis bajas (por debajo de $20 \mathrm{mg}$ ), con frecuencia el paciente presenta síntomas de abstinencia a opiáceos (SAO) ligeros como malestar general, irritabilidad e insomnio que pueden dificultar la desintoxicación. Estos síntomas durante la reducción, pueden complicar la retirada total de metadona, ya sea porque el paciente demande la paralización del descenso o una subida de la dosis, o incluso porque realice nuevos consumos de metadona ilegal o de heroína, recayendo de nuevo en el consumo de opiáceos ilegales. Para disminuir este riesgo, en ocasiones se utiliza en el medio ambulatorio, al llegar a dosis bajas de metadona, ayuda farmacológica (dextropropoxifeno o alfa2-agonistas junto con ansiolítico-hipnóticos) y en ocasiones es necesario solicitar ingreso en unidades de desintoxicación hospitalaria (UDH).

En las UDH la tendencia actual, en el tratamiento farmacológico de la desintoxicación es utilizar dosis decrecientes de agonistas (metadona) o bien alfa2agonistas ${ }^{5,6}$.
Para minimizar los problemas de la desintoxicación de metadona en las unidades de desintoxicación hospitalaria (UDH) es práctica habitual solicitar una dosis máxima de metadona (alrededor de $40 \mathrm{mg}$ ) al ingreso para realizar la desintoxicación. Sin embargo esto está escasamente reseñado en la literatura ${ }^{4,7,8}$

En la práctica diaria, esto no siempre es posible, bien por el tiempo necesario para la disminución gradual ambulatoria, ya sea por partir el paciente de dosis muy elevadas de metadona, o por precisar la desintoxicación en un corto período de tiempo por diversos motivos, o bien por aparecer sintomatología abstinencial durante la misma, que ralentiza o incluso puede llegar a impedir la disminución de dosis ambulatoria.

En nuestra experiencia clínica así ocurre en diversas ocasiones, por lo que empezamos a realizar desintoxicaciones de metadona sin valorar previamente la dosis de partida. Por otra parte la presión asistencial y la experiencia clínica nos señalaba que continuar con disminución gradual de metadona durante el ingreso en UDH, manteniendo al paciente varios días después de llegar a dosis 0 en la Unidad (dado que en esos días aparecían síntomas abstinenciales ligeros pero molestos) suponía estancias demasiado prolongadas en la UDH.

Por ello, optamos por suspender la metadona desde el primer día y utilizar alfa2-agonistas, que habían mostrado su utilidad en el síndrome de abstinencia, tanto en régimen hospitalario como ambulatorio. El empleo de clonidina ${ }^{9}$, tiene un papel fundamental al reducir la hiperactividad noradrenérgica que aparece en el síndrome de abstinencia (SAO) y bloquear la sintomatología precipitada por la naltrexona ${ }^{10-12}$. La utilización de naltrexona se realiza a partir de séptimo día de ingreso y de abstinencia de metadona, tras verificar la abstinencia con el test de naloxona ${ }^{13}$. El alta se produce entre el octavo y décimo día de ingreso.

El objetivo del presente trabajo es comunicar nuestra experiencia en la desintoxicación de metadona de pacientes que son dependientes de opiáceos en mantenimiento con metadona (PMM) en UDH, sin que previamente se haya solicitado a los dispositivos ambulatorios una dosis máxima de metadona previo al ingreso en UDH, independientemente de la dosis de metadona que reciben.

\section{MATERIAL Y MÉTODOS}

Desde 1985 funciona en el Servicio de Psiquiatría del Hospital Ramón y Cajal la Unidad de Desintoxicación Hospitalaria (UDH). Consta de dos camas dentro del Servicio de Psiquiatría general para desintoxicación de los pacientes remitidos desde los dispositivos comunitarios ambulatorios de tratamiento de drogode- 
pendientes de la Comunidad de Madrid (CAD y CAID). Estos centros son los que realizan la indicación de desintoxicación hospitalaria, y al alta se les remite de nuevo los pacientes donde se integran en tratamientos de deshabituación.

La población del estudio se compone de los 22 pacientes con diagnóstico de dependencia de opiáceos en PMM que desde junio 2005 hasta junio 2007, fueron remitidos a la UDH para desintoxicación de dicha sustancia.

Para la valoración de los datos se utiliza una entrevista semiestructurada que contempla los datos socidemográficos y relativos a su historia de consumo ${ }^{14,15}$.

Mediante técnicas descriptivas (media aritmética y desviación estándar en variables cuantitativas, y frecuencias relativas y absolutas en variables cualitativas) se procede a caracterizar la muestra poblacional.

El 79\% de estos adictos son varones, con una edad media de 36,24 años (D.S.=4,28). El tiempo medio de tratamiento con metadona fue de 8,75 años (D.S.= $5,94)$ y la dosis media de la misma al ingreso fue de 59 $\mathrm{mg}(\mathrm{D} . \mathrm{S} .=35)$.

Al ingreso se comunica a los pacientes que durante el tratamiento van a tener algunos síntomas de abstinencia, y que va a ser difícil el que consigan en esos días un sueño satisfactorio. Se intenta desmitificar algunos tópicos sobre el síndrome de abstinencia, y se les informa de los posibles síntomas abstinenciales que pueden presentar ${ }^{16}$

Las causas por las que se ha solicitado desintoxicación han sido variadas (ver Tabla 1), algunas en relación con la finalización del programa y el largo tiempo en tratamiento $(n=8)$ y otras con la demanda del paciente, por diversos motivos entre los que se cuentan problemas laborales $(n=2)$ y cambio de domicilio $(n=2)$. Otras causas han estado relacionadas con efectos secundarios a la propia sustancia, tras aumento de dosis, bien tras inducción enzimática $(n=3)$ o por consumo importante de cocaína o benzodiacepinas $(n=7)$.

Tabla 1. Motivos de solicitud de Desintoxicación

\begin{tabular}{|c|c|c|}
\hline & $\mathbf{N}$ & $\%$ \\
\hline Finalización programada del tratamiento & 8 & 36.36 \\
\hline \multicolumn{3}{|l|}{ Demanda del paciente } \\
\hline Motivos laborales & 2 & 9.09 \\
\hline Cambio de domicilio & 2 & 9.09 \\
\hline \multirow{3}{*}{$\begin{array}{r}\text { Efectos secundarios por aumento de dosis por: } \\
\text { Inducción enzimática } \\
\text { Consumo de cocaína/BZD }\end{array}$} & & \\
\hline & 3 & 13.63 \\
\hline & 7 & 31.81 \\
\hline Total & 22 & 100 \\
\hline
\end{tabular}

\section{RESULTADOS}

Del total de los 22 pacientes remitidos a la UDH para realizar la desintoxicación de metadona, lo realizan con éxito el 95\% de ellos. Sólo 1 paciente solicitó el alta voluntaria a las $24 \mathrm{~h}$ del ingreso.

La dosis media de metadona fue de $59 \mathrm{mg}$ (d.s.=35), con un rango que osciló entre 15 y $135 \mathrm{mg}$.

El tratamiento de desintoxicación se inició el primer día del ingreso, con la suspensión de la metadona, que ya no se administraba en la Unidad. A las $24 \mathrm{~h}$ de la última toma del agonista se pautaba clonidina, a dosis media de 0,225 mg/día repartidos en tres tomas, para continuar al día siguiente con una dosis de media de $0,45 \mathrm{mg}$ al día repartida en tres tomas $(0,15-0,15-0,15$ $\mathrm{mg}$ ) hasta el $6-7^{\circ}$ día de ingreso. El séptimo día se inicia la inducción con naltrexona, previo la realización del test de naloxona (0.8 mgr s.c.), iniciándose la suspensión gradual de clonidina en dos días.

El 55\% de los pacientes precisaron medicación coadyuvante para molestias álgicas-musculares que fueron tratadas exitosamente con ibuprofeno. De forma preventiva se pauto tratamiento con omeprazol.

El 30\% refirió diarreas matutinas que no requirieron tratamiento farmacológico.

La tensión arterial en todos ellos se mantuvo con escasas oscilaciones alrededor de 110/70, volviendo a sus niveles habituales tras la suspensión de la clonidina.

Todos los pacientes recibieron alguna dosis concomitante de clonacepan, con un rango que osciló de 1 $\mathrm{mg}$ a $6 \mathrm{mg}$ al día. Esta dosis no dependió de la dosis de metadona sino del consumo concomitante de benzodiacepinas, cocaína o/y alcohol. En 5 pacientes se asoció anticomiciales (oxcarbamacepina o gabapentina) por el consumo concomitante de benzodiacepinas.

El insomnio fue el mayor problema presentado durante la desintoxicación, apareciendo en el $80 \%$ de los pacientes, y siendo de mayor intensidad a partir de $4^{\circ}-6^{\circ}$ día de ingreso. Consistía principalmente en un insomnio de conciliación y medio que era mal tolerado por el paciente. Por ello se prescribió en la mitad de los pacientes antipsicóticos sedantes a bajas dosis.

Los efectos secundarios presentados por los pacientes (ansiedad, insomnio, dolores musculares, etc.) no se relacionaron con la dosis de metadona, sino principalmente con el consumo concomitante de otras sustancias.

La estancia media de los 21 pacientes que realizan la desintoxicación fue de 10,32 días (con un mínimo de 8 días y un máximo de 12 días). 
Las diversas dosis de metadona no supuso más tiempo de estancia, un SAO más marcado, un mayor número de altas voluntarias, ni un mayor uso de fármacos, como podríamos esperar.

\section{DISCUSIÓN}

Durante años mayoritariamente se ha realizado en las UDH desintoxicaciones de heroína. En los últimos años ha cambiado el perfil de la demanda, y se remite desde los dispositivos ambulatorios a la UDH adictos a otras sustancias para desintoxicación ${ }^{17}$. Por ello, en los últimos años las desintoxicaciones de heroína son escasas, y lo más común son las demandas de desintoxicación de cocaína, alcohol y/o benzodiacepinas en PMM. Es recientemente cuando se nos demanda desintoxicación de metadona.

La pauta de desintoxicación presentada ha permitido que a la mayoría de los pacientes en PMM a los que se les solicita la desintoxicación, la realicen, sin previamente haber realizado una disminución gradual ambulatoria de la sustancia, tal y con se exige habitualmente en otros centros. Esta desintoxicación gradual no siempre puede realizarse, debido al tiempo requerido y en muchas ocasiones porque no es tolerada por el paciente, que solicita un aumento de dosis precipitado en muchas ocasiones por ansiedad y SAO. Además supone el problema añadido en nuestro medio del ajuste de fechas entre la disponibilidad del dispositivo y la dosis adecuada del paciente, que no siempre se alcanza correctamente.

Llama la atención la escasa literatura que existe sobre la desintoxicación de metadona en UDH. Existe y muy abundante sobre desintoxicación rápida, con inducción rápida de naltrexona, en régimen de UVI ${ }^{18,19}$, pero es muy escasa sobre las pautas de desintoxicación en UDH, y a nivel ambulatorio son muy antiguas ${ }^{3,7}$.

La diversa dosis de metadona no supuso más tiempo de estancia, un SAO más marcado, un mayor número de altas voluntarias, ni un mayor uso de fármacos, como podríamos esperar. A pesar de que no resultó significativo dado el escaso número de pacientes, la práctica clínica nos señala que estos factores dependen principalmente del consumo de alcohol, benzodiacepinas y/o cocaína.

Existen numerosos estudios muy críticos con la desintoxicación de agonistas, refiriendo que en la deshabituación son frecuentes las recaídas y la vuelta al tratamiento con metadona ${ }^{20,21}$. Esto sin embargo no es el objeto de nuestro debate. Nuestro debate se centra en como podemos realizar la desintoxicación de metadona en UDH, una vez que se ha decidido realizarla. Y no podemos olvidar que esta desintoxicación es un procedimiento que surge actualmente, y en más de la mitad de los casos es solicitado por el propio paciente, que exige su realización. Es previsible que en un futuro esta demanda continúe creciendo y será la realización de estudios rigurosos los que nos indiquen las pautas a seguir ${ }^{22}$.

\section{CONCLUSIONES}

El aumento de adictos a opiáceos en tratamiento en metadona ha supuesto en nuestro medio un aumento de la demanda de desintoxicación a dicha sustancia. La desintoxicación de metadona sin necesidad de disminuir la dosis hasta un umbral determinado como es práctica habitual en las unidades de desintoxicación hospitalaria (UDH), que solicitan una dosis máxima de metadona (alrededor de $40 \mathrm{mg}$ ) se muestra en este trabajo preliminar.

En el se muestra la experiencia en 22 pacientes que en los últimos dos años realizan desintoxicación de metadona sin solicitar a los centros remitentes dosis límite máxima para su ingreso. Reciben durante la desintoxicación tratamiento con clonidina y benzodiacepinas (ajustada la dosis de éstos fármacos según el consumo concomitante de alcohol, benzodiacepinas o cocaína) y analgesia no opiácea. Al séptimo día se administra $50 \mathrm{mg}$ de naltrexona. De estos adictos, 21 realizaron la desintoxicación de forma adecuada. La dosis de metadona no modificó la sintomatología abstinencial.

A pesar de nuestra corta experiencia y la necesidad de realizar estudios más rigurosos, la clínica nos indica que no es necesario la limitación de dosis para realizar la desintoxicación de metadona en UDH, precisándose continuar la investigación al respecto.

\section{REFERENCIAS}

1. Agencia Antidroga. Consejería de Sanidad y Servicios Sociales. Comunidad de Madrid. Memoria 1998.

2. Agencia Antidroga. Plan Regional sobre Drogas. Comunidad de Madrid. Memoria 2005.

3. Senay EC, Dorus W, Goldberg F, Thornton W. Withdrawal from methadone maintenance. Rate of withdrawal and expectation. Arch Gen Psychiatry 1977; 34: 361-367.

4. Fernández Miranda JJ, Gutiérrez Cienfuegos E, Bobes García J. Tratamientos de mantenimiento con metadona. En: Manual de evaluación y tratamiento de drogodependencias. Eds: Julio Bobes García, Miguel Casas Brugué, Miguel Gutierrez Fraile. Psiquiatría Editores SL 2003; 232-257. 
5. Stine SM, Kosten TR. Use of drug combinations in treatment of opioid withdrawal. J Clin Psychopharmacol 1992; 12: 203-209.

6. Fernández Miranda JJ. Efectividad de los programas de mantenimiento con metadona. Una revisión de los resultados de los estudios de evaluación. Med Clin 2001; 116: 150-154.

7. Strang J, Gossop M. Comparison of linear versus inverse exponential methadone reduction curves in the detoxification of opiate addicts. Addict Behav 1990; 15: 541-547.

8. Parrino MW. Manual de tratamiento de metadona. Grup Igia. Barcelona; 1997.

9. Gold MS, Pottash AC, Sweeney DR, Kleber HD. Opiate withdrawal using clonidine: A safe, effective and rapid non opiate treatment. JAMA 1980; 243: 343-346.

10. Charney DS Heninger GR, Kleber HD. The combined use of clonidine and naltrexone as a safe, rapid and effective treatment of abrupt withdrawal from methadone. Am J Psychiatry 1986; 143: 831-837.

11. Kleber HD. Detoxification from narcotics. In Lowinson JH, Ruiz P (eds): Substance abuse: Clinical problems and perspectives. Maryland. Williams \& Wilkins. 1991; 24: 317-338.

12. Senft AR. Experience with clonidine-naltrexone for rapid opiate detoxification. J Subs Abuse Treatment 1991; 8: 257-259.

13. Janiri L, Mannelli P, Persico AM, Serretti A, Tempesta E. Opiate detoxification of methadone maintenance patients using lefetamine, clonidine and buprenorphine. Drug Alcohol Depend 1994; 36: 139-145.

14. Baca-García E, García A, Ochoa E. Historia Psiquiátrica Estructurada en la Interconsulta Hospitalaria a
Toxicomanías/Sida. Revista Española Drogodependencias 1999; 2481: 60-70.

15. Baca-García E, Madoz-Gúrpide A. Protocolos de recogida de datos en una unidad de toxicomanías con "Clínica de naltrexona". En: Ochoa Mangado E. Antagonistas opiáceos en las dependencias. Clínica de la Naltrexona. Col. Psiquiatría 21. Dir. Prof. Sáiz Ruiz J. Barcelona: Ed. Ars Medica. Psiquiatría Editores SL; 2001.

16. Gossop M. The development of a Short Opiate Withdrawal Scale (SOWS). Addict Behav. 1990; 15: 487-490.

17. Olmos Espinosa R, Madoz-Gúrpide A, Ochoa E. Evolución al año de los adictos a opiáceos que realizan desintoxicación en UDH y continúan tratamiento ambulatorio. Adicciones 2001; 13: 173-178.

18. Hensel M. Safety, efficacy, and long-term results of a modified version of rapid opiate detoxification under general anaesthesia: a prospective study in methadone, heroin, codeine and morphine addicts. Acta Anaesthesio Scandinava 2000; 44: 326-333.

19. Prieto IJ, Poyo-Guerrero R, Fernandez R, Ochoa E. Ultrarapid high-dose methadone detoxification. Psychopharma-cology 2003; 165: 430.

20. Magura S, Rosenblum A. Leaving methadone treatment: lessons learned, lessons forgotten, lessons ignored. The Mount Sinai Journal of Medicine 2001; 68: 62-74.

21. Masson $\mathrm{CL}$, Barnett $P G$, Sees $\mathrm{KL}$, Delucchi $\mathrm{KL}$, Rosen A, Wong W, Hall SM. Cost and cost-effectiveness of standard methadone maintenance treatment compared to enriched 180-day methadone detoxification. Addiction 2004;99:718-726.

22. Fernández Miranda JJ, Torrens M (Eds). Monografía opiáceos. Adicciones 2005; 17. 
